\title{
A head and neck hyperthermia applicator: theoretical antenna array design
}

Margarethus M Paulides, Jurriaan F Bakker, Adrianus PM Zwamborn, Gerard C van Rhoon

Erasmus MC - Daniel den Hoed Cancer Center, Department of Radiation Oncology, Section Hyperthermia PO box 5201, NL-3008 AE, Rotterdam, The Netherlands

Tel.no. : +31 104391676

Fax.no. : +31 104391022

E-mail: M.Paulides@ErasmusMC.nl

August 22, 2007

Running title: "H\&N applicator: antenna array investigation" 


\begin{abstract}
Purpose: Investigation into the feasibility of a circular array of dipole antennas to deposit RF-energy centrally in the neck as a function of: 1) patient positioning, 2) antenna ring radius, 3) number of antenna rings, 4) number of antennas per ring and 5) distance between antenna rings.

Materials and Methods: Power absorption (PA) distributions in realistic, head and neck, anatomy models are calculated at $433 \mathrm{MHz}$. Relative PA distributions corresponding to different set-ups were analysed using the ratio of the average PA (aPA) in the target and neck region.

Results: Enlarging the antenna ring radius from $12.5 \mathrm{~cm}$ to $25 \mathrm{~cm}$ resulted in a $\sim 21 \%$ decrease in aPA. By changing the orientation of the patients with respect to the array an increase by $\sim 11 \%$ was obtained. Increase of the amount of antenna rings led to a better focussing of the power $(1 \rightarrow 2 / 3: \sim 17 \%)$. Increase of the distance between the antenna rings resulted in a smaller (more target region conformal) focus but also a decreased power penetration.

Conclusions: A single optimum array setup suitable for all patients is difficult to define. Based on the results and practical limitations a setup consisting of two rings of six antennas with a radius of $20 \mathrm{~cm}$ and $6 \mathrm{~cm}$ array spacing is considered a good choice providing the ability to heat the majority of patients.
\end{abstract}

Key words: Hyperthermia, SAR calculations, power absorption, antenna array, head and neck tumours.

\title{
1 Introduction
}

Hyperthermia (HT) applied to advanced, unresectable, head and neck $(\mathrm{H} \& \mathrm{~N})$ carcinomas, and their lymph node metastases, has a high potential to improve the results of currently existing treatment strategies [1] [2] [3] [4] [5]. Hereto equipment is warranted that enables application of an adequate heating pattern to this region. To the best of the authors knowledge, such equipment is available only for heating depths up to $4 \mathrm{~cm}$. Further, these superficial applicators provide only limited possibilities to adjust the specific absorption rate (SAR) pattern. Since we aim at heating both the primary tumour and lymphnode metastases, we need to design a specific H\&N HT applicator to achieve an optimal treatment. This novel applicator must provide a high degree of control of the SAR deposition pattern that should result in a high quality of the HT-treatment as shown by Wust et al. [6].

In earlier work [7] [8] we have investigated the dependence of the SAR pattern on a selection of basic design criteria: such as frequency, number of antenna elements and anatomy. In these studies we demonstrated that due to circular symmetry of the neck anatomy a circular array of antenna-elements is to be preferred. Further it was shown that the optimal frequency is highly dependent on the target volume to be treated. Based 
on the expected target volumes for $\mathrm{HT}$ in the $\mathrm{H} \& \mathrm{~N}$ region, we selected $433 \mathrm{MHz}$ as most preferable frequency. This frequency provides a good focussing of the energy and relatively low-priced equipment is available for this frequency. Finally it was demonstrated that six to eight antennas on a circular ring are sufficient to obtain focussed central heating for neck diameters up to $12 \mathrm{~cm}$. For neck diameters larger than $12 \mathrm{~cm}$ the focus intensity decreases but in general sufficient energy is deposited at depth.

In this paper we report on the results of a theoretical study that essentially is a followup of these earlier studies. In the present theoretical investigation the focus firstly is on selection of a more realistic antenna arrangement, i.e. a single optimal and easily applicable antenna ring radius for all neck anatomies. This is carried out by numerically investigating the SAR focussing as a function of antenna ring radius, i.e. the patient-antenna distance is increased. Secondly, the aim is to investigate the opportunities that multiple antenna rings provide for axial SAR focussing. The investigation is performed by calculation of $3 \mathrm{D}$ power absorption (PA) distributions in two realistic head models. The goal is to develop an applicator that provides the flexibility to efficiently heat superficial target areas as well as more deeply located target volumes. Further, it should enable steering away from critical organs avoiding toxicity [9]. Again, we assumed that the ability to obtain a high PA at the center of the neck will be the most challenging and difficult problem to solve. As a consequence, we consider that if adequate heating can be obtained for centrally located target volumes then phase and amplitude PA steering can effectively be used to heat lateral target volumes. Therefore, in this theoretical study, we have focussed on investigating the dependence of the PA at the center of the neck as a function of the arrangement of a multi-ring antenna array.

\section{Materials and Methods}

The antenna arrangement that provides the optimum PA pattern, and the highest efficiency, varies between patients, tumour locations and tumour volumes. Further, the location and orientation of the patient with respect to the antenna array can have a major influence. Investigating all above mentioned variables and their combined effects is obviously a vast task. To restrict the number of simulations, we split our investigation into two series of calculations with growing complexity. Parameters of interest for each series of investigations were: 
- First series

- Patient positioning ("RT position" vs "HT position")

- Antenna ring radius ("R")

- Second series

- Number of antenna rings

- Number of antenna elements per antenna ring

- Distance between the outer antenna rings ("D")

The aim of the first series of calculations was to explore the influence of the antenna ring radius, aiming to find a single diameter for all anatomy sizes. Hereto we started with a setup of eight antenna elements that are equidistantly space on an imaginary ring round the neck, and investigated the influence of the radius of the antenna ring on the PA pattern. Simultaneously, we investigated the effect of patient positioning. From the first series of calculations we selected the antenna ring radius and patient positioning for further investigations. The second series of calculations was dedicated to the number of antenna rings, the amount of antennas per ring and the distance between these antenna rings.

\subsection{Anatomy and antenna arrangements}

Previously, we examined CT-scans of eight patients that underwent radiotherapy (RT) at various locations in the head and neck region and we determined the minimum and maximum diameter of the neck [7]. These results where used to select two patients with respectively the smallest (radius $\sim 4.95 \mathrm{~cm}$ : "small patient model") and largest neck (radius $\sim 6.75 \mathrm{~cm}$ : "large patient model") from this subgroup. The small patient had a carcinoma in the larynx and the large patient a nasopharynx carcinoma. A CT-scan of these patients was used to segment two head and neck tissue models. These patients are assumed to represent the extends of the group of patients in terms of heating in the neck, i.e. results are expected to be representative for the whole group of patients. Both models contain over ten tissue types (Table 1). The CT-scans contained anatomy information from the shoulders up to the nose for the small patient and from the shoulders up to the crest for the large patient. By comparing the PA patterns in patient models with and without an artificial extension we found that the truncations affect only the PA values within $\sim 2 \mathrm{~cm}$ from the truncation. Figure 1 shows the tissue distributions of both anatomy models.

In [8] we found that $433 \mathrm{MHz}$ is within the optimum range of frequencies for an eight dipole antenna setup and that the array should be rotated $22.5^{\circ}$ with respect to the chin to

avoid hotspots at the chin. In Figure $4 \mathrm{a}$ we present the setup to investigate the influence of 
Table 1: Dielectric properties at $433 \mathrm{MHz}$ of the tissues types corresponding to the two head models $\left(\mu_{r}=1.0\right)$.

\begin{tabular}{|c|c|c|}
\hline Tissue type & $\epsilon_{r}$ & $\sigma_{e f f}[\mathrm{~S} / \mathrm{m}]$ \\
\hline Demineralized water & 78 & 0.04 \\
\hline Trachea & 1 & 0.00 \\
\hline Fat & 6 & 0.04 \\
\hline Muscle & 57 & 0.81 \\
\hline Bone $_{\text {Cortical }}$ & 13 & 0.09 \\
\hline Air bubble & 1 & 0.00 \\
\hline Lung & 1 & 0.00 \\
\hline Tooth & 13 & 0.09 \\
\hline Cartilage & 45 & 0.60 \\
\hline Brain $_{\text {Grey }}$ & 57 & 0.75 \\
\hline Brain $_{\text {White }}$ & 42 & 0.45 \\
\hline Skin $_{W e t}$ & 46 & 0.68 \\
\hline Gland & 61 & 0.89 \\
\hline Eye & 58 & 1.01 \\
\hline PEC & 1 & $1 * 10^{6}$ \\
\hline
\end{tabular}

variation of the antenna ring radius $R$ on the absorption pattern in the neck. A minimum radius of $12.5 \mathrm{~cm}$ was used to ensure that both anatomies are located in the far field of the dipoles $(\sim \lambda / 2)$. This radius was enlarged to $25 \mathrm{~cm}$ in steps of $2.5 \mathrm{~cm}$ to obtain the required minimum distance between the tissue surface and the antenna elements. Simultaneously, we investigated the effect of a ten degrees rotation of the array with respect to the patient around the x-axis (left $\rightarrow$ right): "RT position" vs "HT position" (see Figure 1 and 2). The RT position conforms to the RT treatment position and the HT position is considered optimal for a HT treatment. We chose a ten degrees rotation (around the x-axis) because this leads to a smaller neck cross-section and aligns the spinal column of the patient with the central axis of the applicator.

Using the selected antenna ring radius and anatomy rotation angle, the second series of calculations addressed the potential of multiple antenna rings. As a starting point we used setups with six and eight antennas and investigated the benefit of setups consisting of two or three antenna rings. We further changed the distance between the outer antenna rings ( $\mathrm{D}$ in Figure 2) stepwise from 0 to $10 \mathrm{~cm}$. With multiple rings at a suitable distance we expected to obtain a more axially focussed absorption pattern and axial steering possibilities. In practice, the maximum amount of antennas is restricted to twelve since this amount of 150 Watt amplifiers, operating at $433 \mathrm{MHz}$, is available in our unit. This 


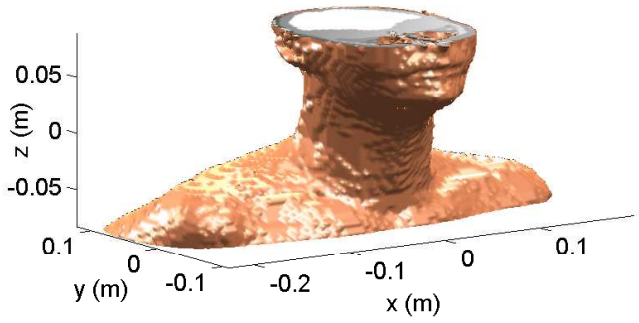

a) Small patient model

(3D view)

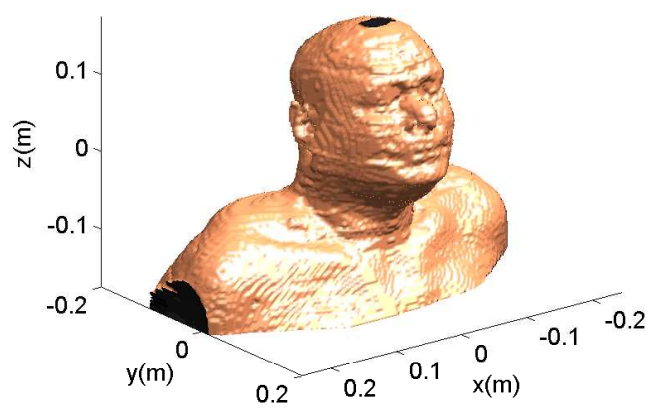

c) Large patient model

(3D view)

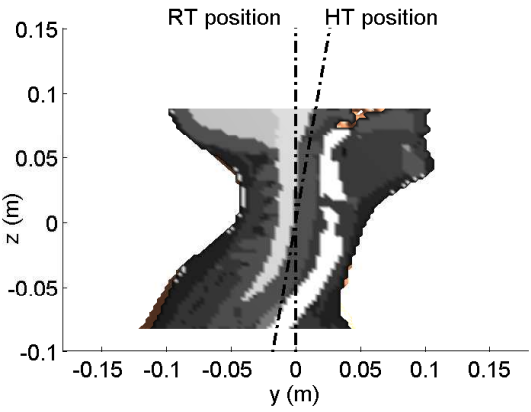

b) Small patient model (Sagittal cut, $\mathrm{x}=0$ )

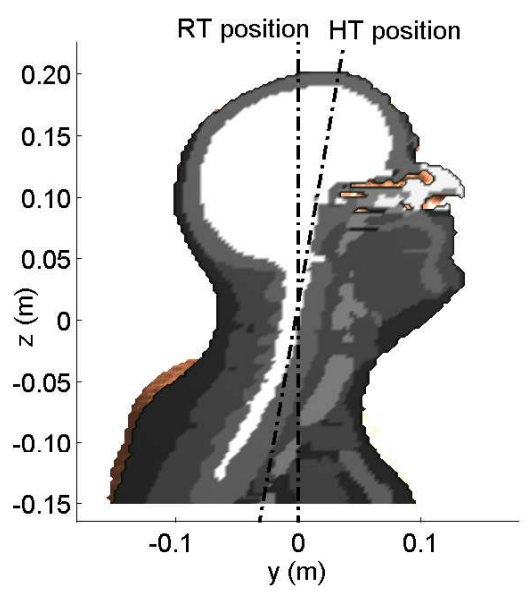

d) Large patient model (Sagittal cut, $\mathrm{x}=0$ )

Figure 1: 3D view $(\mathrm{a}, \mathrm{c})$ and sagittal cuts $(\mathrm{b}, \mathrm{d})$ of the tissue distribution of the two head models. b) and d) show the RT position and an indication of the HT position (10 rotation).

leads to setups of two rings of four (" $2 \times 4 ")$ and six (" $2 \times 6 ")$ antennas and three rings of four antennas ("3x4"). Nevertheless, two rings of eight antennas ("2x8") arrangement was investigated as well to quantify the relevance of a possible extension to more antennas and amplifiers. The arrangements are schematically shown in Figure 2. Note that we applied a symmetrical rotation of the setups around the z-axis with respect to the chin. Further note that the $2 \mathrm{x} 4$ arrangement with $0 \mathrm{~cm}$ distance is equal to a $1 \mathrm{x} 8$ arrangement. 


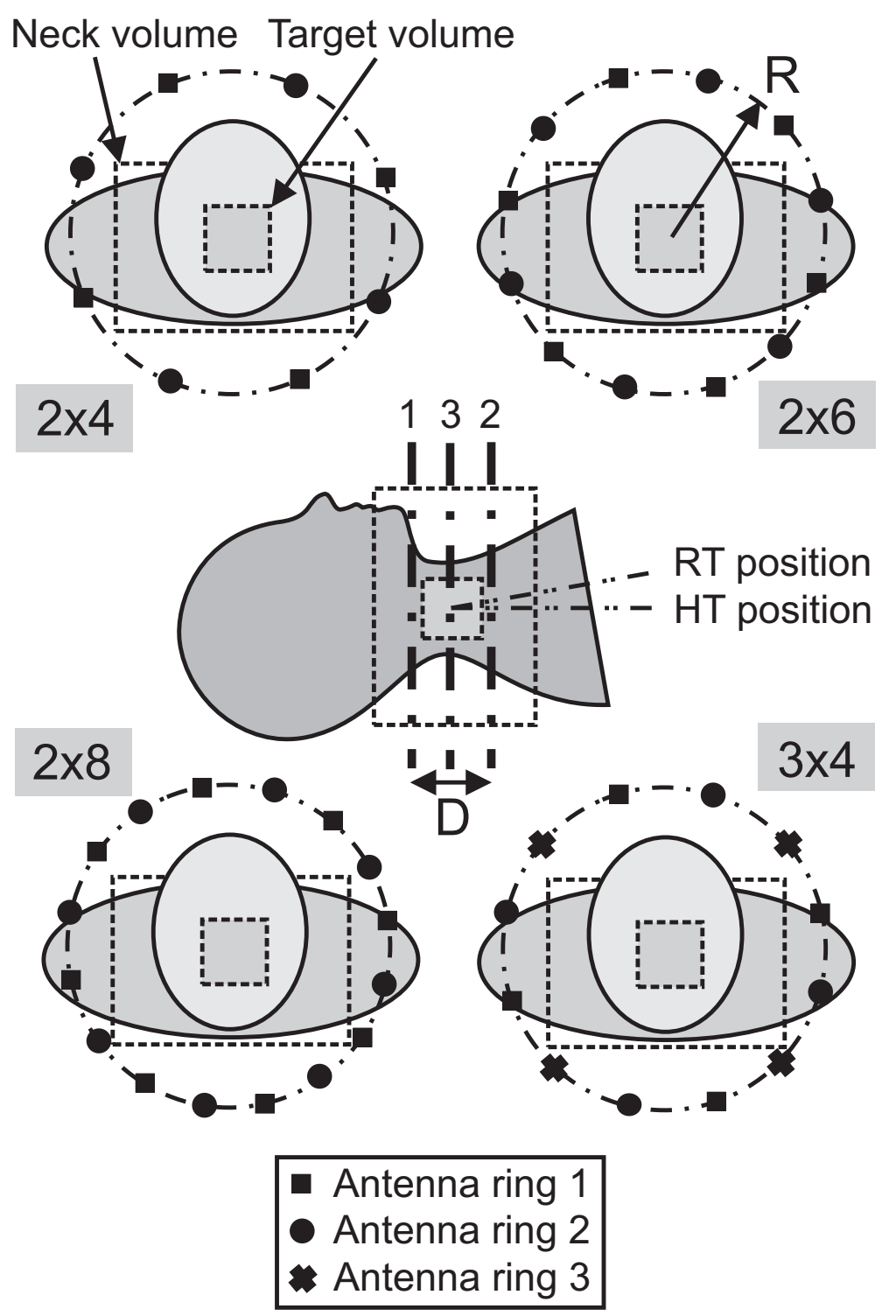

Figure 2: Multiple antenna ring arrangements and a schematic sagittal view in HT position. 


\subsection{Electromagnetic modelling}

We predicted PA distributions in both patient models by computing the electromagnetic field using a finite-difference time-domain (FDTD) based program ${ }^{1}$. PA was defined as:

$$
P A=\frac{\sigma_{e f f}|\vec{E}|^{2}}{2}, \quad\left(W / m^{3}\right)
$$

where $\sigma_{e f f}$ is the effective electrical conductivity $(\mathrm{S} / \mathrm{m})$ and $\vec{E}$ is the complex electric field vector. Note that the PA values differ only by the tissue-dependent mass density from specific absorption rate (SAR) values. Mur's second-order absorbing-boundary conditions $(\mathrm{ABC})$ and additional extra voxels of exterior are applied to eliminate the influence of reflections at the boundaries of the computational domain. The dimensions of the, in total $2.5 \mathrm{M}$, voxels were $2.9,2.9$ and $2.5 \mathrm{~mm}(\Delta<\lambda / 25)$. The dielectric properties of the tissues (Table 1) were assumed isotropic and were found using a parametric model by Gabriel et al. [12]. The dielectric properties of the trachea are chosen equal to those of air. The properties of the de-mineralized water (salinity $=0 \mathrm{~g} / \mathrm{l}, \mathrm{T}=20^{\circ} \mathrm{C}$ ) in the waterbolus are found in a publication of Stogryn [13] and one of the ESHO taskgroup comittee [14]. Dipoles were approximated by two straight conducting wires (PEC material, Table 1) with a cross-section of one voxel $(2.9 \mathrm{~mm} \times 2.9 \mathrm{~mm})$. The length of the dipole antennas $\left(L_{d i p}\right)$ was chosen such that they are resonant at $433 \mathrm{MHz}$ in water, i.e. $3.75 \mathrm{~cm}$. Excitation was obtained by a suppressed electric field at the gap between both wires. For the investigations we always excited the sources with equal phases and amplitudes since this is expected to result in a central absorption focus. In the entire study, phase and amplitude optimization has been omitted because little improvements are expected for this symmetrical antenna arrangement and almost symmetrical patient anatomy in the axial plane [7]. For the setup with three rings this approach introduced a small error of 9 degrees phase mismatch between the middle and outer rings for central steering. The result of such a small phase difference, however, has a minor impact on the aPA ratio as it only slightly affects the focus at its axial extends that are not within the target area. The waterbolus is modelled by an infinite de-mineralized water environment since investigation of the effects of the waterbolus was beyond the scope of this investigation.

\subsection{Evaluation}

Simulated PA patterns were analysed qualitatively by comparing visualizations of crosssections through the origin of the applicator, i.e. through $\mathrm{x}=0, \mathrm{y}=0$ and $\mathrm{z}=0$. These plots visualize PA values that are normalized to the average value in the neck region. For quantitative evaluation we defined the average PA ratio that quantifies the average

\footnotetext{
${ }^{1}$ FDTD program developed at TNO, The Hague, The Netherlands. [10] [11]
} 
amount of power that is absorbed in the target region with respect to the average amount in the neck:

$$
\text { aPA ratio }=\frac{\overline{P A}_{t g t}}{\overline{P A}_{n c k}}
$$

where $\overline{P A}_{t g t}$ is the mean power absorption value in the target region and $\overline{P A}_{n c k}$ is the mean power absorption value in the neck region. The aPA ratio effectively quantifies the global shape of the PA pattern. For this analysis we introduced a centrally located hypothetical target volume with realistic content in both anatomies as shown in Figure 2 . Target volumes of $4 \mathrm{~cm} \mathrm{x} 4 \mathrm{~cm} \mathrm{x} 4 \mathrm{~cm}\left(0.064 \mathrm{dm}^{3}\right), 5 \mathrm{~cm} \times 5 \mathrm{~cm} \times 5 \mathrm{~cm}\left(0.125 \mathrm{dm}^{3}\right)$ and $6 \mathrm{~cm}$ $\mathrm{x} 6 \mathrm{~cm} \mathrm{x} 6 \mathrm{~cm}\left(0.216 \mathrm{dm}^{3}\right)$ are investigated because the aim is to treat advanced tumours. Results are shown only for $5 \mathrm{~cm}$ x $5 \mathrm{~cm}$ x $5 \mathrm{~cm}$ since observed phenomena were similar for all three target volume sizes. The height of the neck volume was $16 \mathrm{~cm}$ (small anatomy) or $20 \mathrm{~cm}$ (large anatomy) to incorporate the PA values up to the chin and shoulders. High local PA values ("PA hotspots") were found at these sites in the previous investigation [8] and these effects are potentially decreased by a proper setup with multiple antenna rings. This larger neck volume means that the aPA values will be higher than in previous publication since more low values are included in the neck volume and as a consequence the average PA in the neck volume will be lower.

\section{Results}

\subsection{First series: ring radius and patient model orientation}

Figure 3 shows a sagittal, transversal and coronal cross-section of the PA distribution in the large patient model corresponding to two setups: 1) one ring of eight dipoles ("1x8") and 2) two rings of six dipoles (" $2 \times 6$ "), both with a radius of $20 \mathrm{~cm}$.

The transversal cross-section of the $1 \times 8$ setup clearly shows a central region of high PA: the central focus. This central focus quite nicely fits in the inner white square: the target region. The sagittal and coronal view confirm this central focus but reveal that the axial focus length extends to outside of the target volume. Further it shows regions of high PA in tissue outside the target region ("PA hotspots") at the chin and shoulders. The locations of these hotspots are indicated because they are poorly visible.

Figure 4 visualizes aPA ratios as a function of applicator ring radius (1x8 setup) for a small and large patient model in RT and HT position. The aPA ratio is found to decrease about $21 \%$ for arrangements with increasing radii. An increasing radius leads to a larger focus length (z-direction) so relatively less power is deposited within the $5 \mathrm{~cm}$ of the target region. The slopes of the curves are dependent on the choice of the neck volume but the shape is comparable for different volumes. A radius of $20 \mathrm{~cm}$ is selected for the clinical applicator so all antennas are sufficiently far away for all patients and for ease of patient 


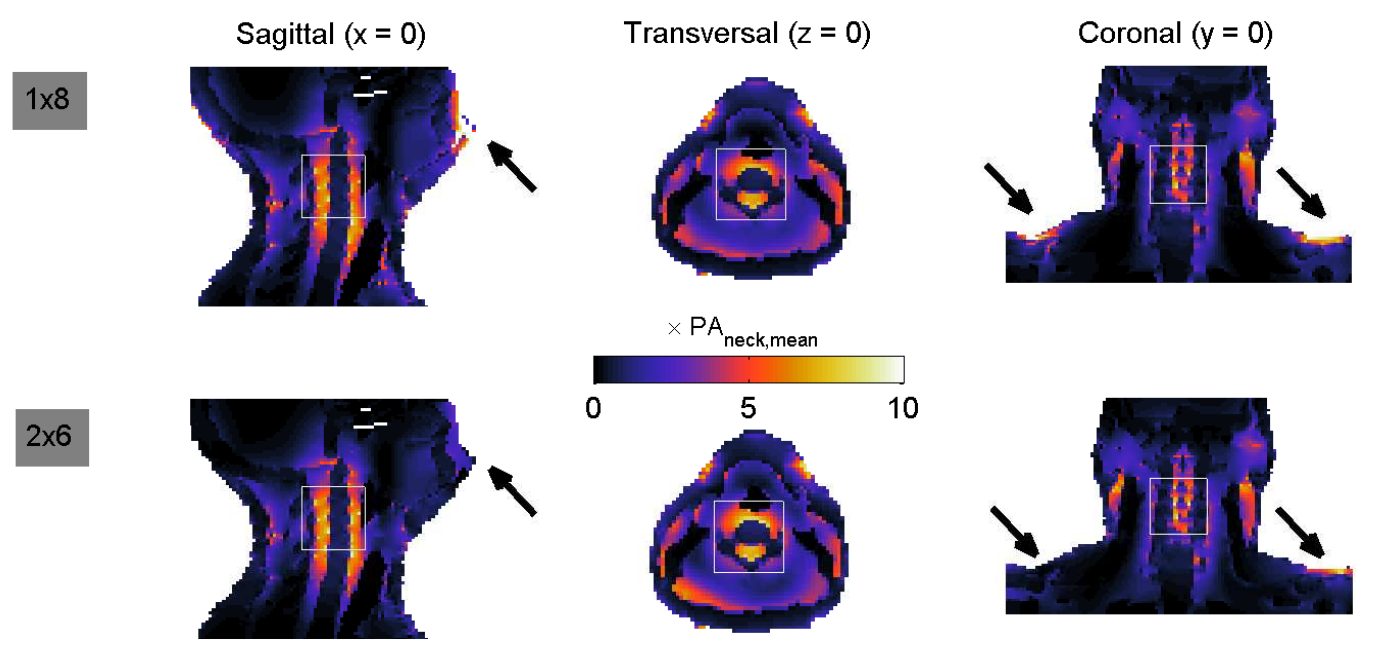

Figure 3: Cross-sections of the PA distributions in the large patient model in HT position for one ring of eight dipoles $(1 \mathrm{x} 8, \mathrm{R}=20 \mathrm{~cm})$ and two rings of six dipoles $(2 \times 6, R=20 \mathrm{~cm}$, $\mathrm{D}=6 \mathrm{~cm})$. Black arrows indicate the locations of PA hotspots $(1 \times 8)$ or corresponding locations where these hotspots are reduced $(2 \times 6)$.

positioning. The figure also shows that the HT position leads to favourable aPA ratios compared to the RT position for the small $(+5 \%)$ and large $(+18 \%)$ patient model. Hence, a PA pattern is obtained with a more pronounced central focus and a lower sensitivity to the neck diameter. This more intense focus is the result of a smaller neck cross-section in HT positioning (perpendicular to the spine, see Figure 1). From these results we chose the HT position as optimum and hence used the HT position and a radius of $20 \mathrm{~cm}$ for further calculations.

\subsection{Second series: number of rings, amount of antennas per ring and ring distance}

When enlarging $\mathrm{D}$ we found for all array arrangements that the central focus becomes smaller in the axial direction and more and more conforms to the target region. When the distance $\mathrm{D}$ becomes too large, the intensity of the focus is hampered by insufficient penetration of the electromagnetic waves due to an increasing path length. Further, the PA hotspots at both shoulders reduce for all arrangements when enlarging D up to maximum $8 \mathrm{~cm}$. These effects are exemplified by Figure 3 that shows PA distributions for $1 \times 8$ and $2 \mathrm{x} 6$ configurations. The main difference between the PA patterns of both setups are the PA hotspots at the chin and shoulders for the $1 \times 8$ setup that are reduced in the $2 \times 6$ setup: 


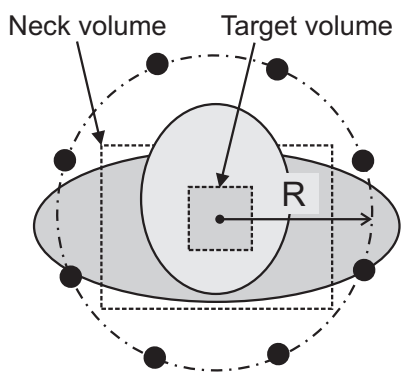

a) Setup

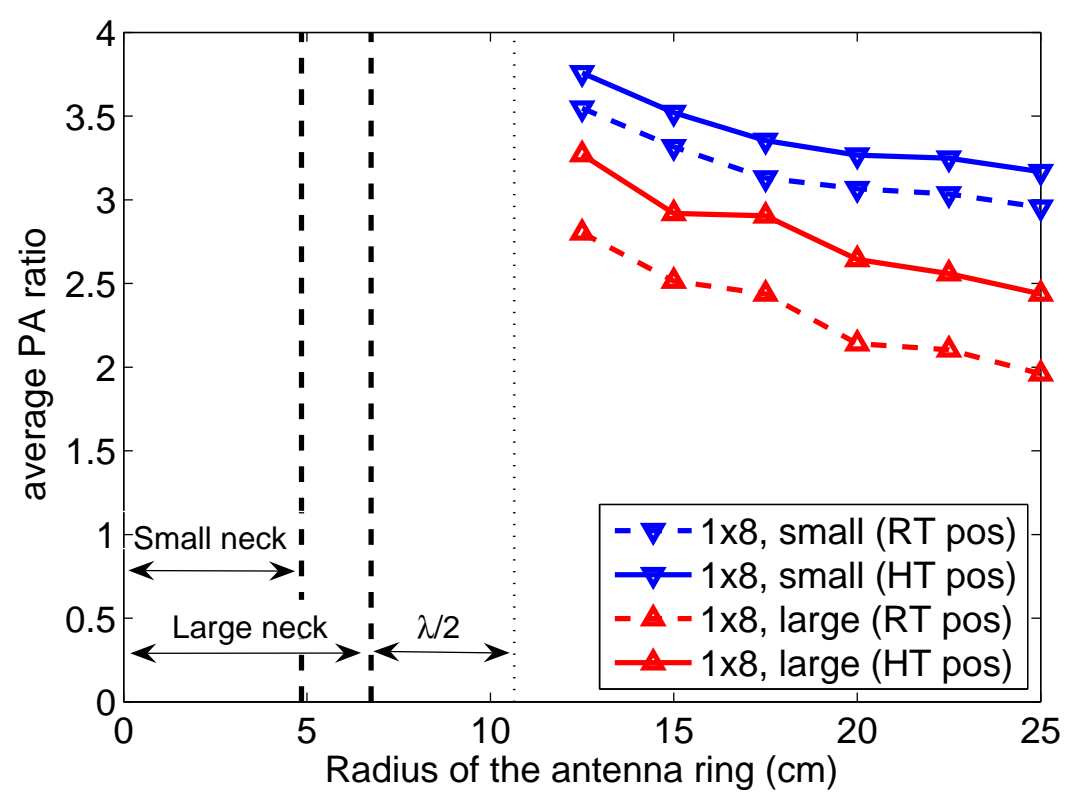

b) Results

Figure 4: Average PA ratios as a function of antenna ring radius for a setup of eight antennas: setup a) and results b). Curves correspond to results for a small and a large patient model in RT and $\mathrm{HT}\left(10^{\circ}\right.$ rotation around the $\mathrm{x}$-axis) position. The average radius of these models and the near field distance $(\lambda / 2)$, where the dipole antennas should not be placed, are indicated.

this effect is indicated by the arrows. For both setups we found a central focus that is modulated by the anatomy distribution. The PA pattern, however, is somewhat more focussed by using two rings, however, it is difficult to notice this effect on the base of PA visualizations.

Figure 5 visualizes the aPA ratios for all antenna arrangements, all distances and both anatomy models. For an increased antenna outer ring distance, it quantifies the previously mentioned effects: an increase of focus but a decrease of intensity at the target region. The figure shows that extending the amount of antenna rings from one to two or three results in $\sim 15 \%$ (small patient) to $\sim 19 \%$ (large patient) more power absorption at depth. Three out of four aPA ratio curves show highest values for the setups with $\mathrm{D}=0 \mathrm{~cm}$. The aPA of these curves, however, is relatively stable until a certain threshold distance where the decrease becomes much steeper. This effect is less prominent for a $3 \times 4$ setup but in this case the third central ring is not moved (see Figure 2). For a $2 \times 8$ setup, maximum focussing is not obtained at $\mathrm{D}=0 \mathrm{~cm}$ because each ring of eight antennas provides optimum PA intensity at the target region on its own but the focus in z-direction conforms best to the target region for ring distances around $4-6 \mathrm{~cm}$. All curves are positively correlated 
Small patient model

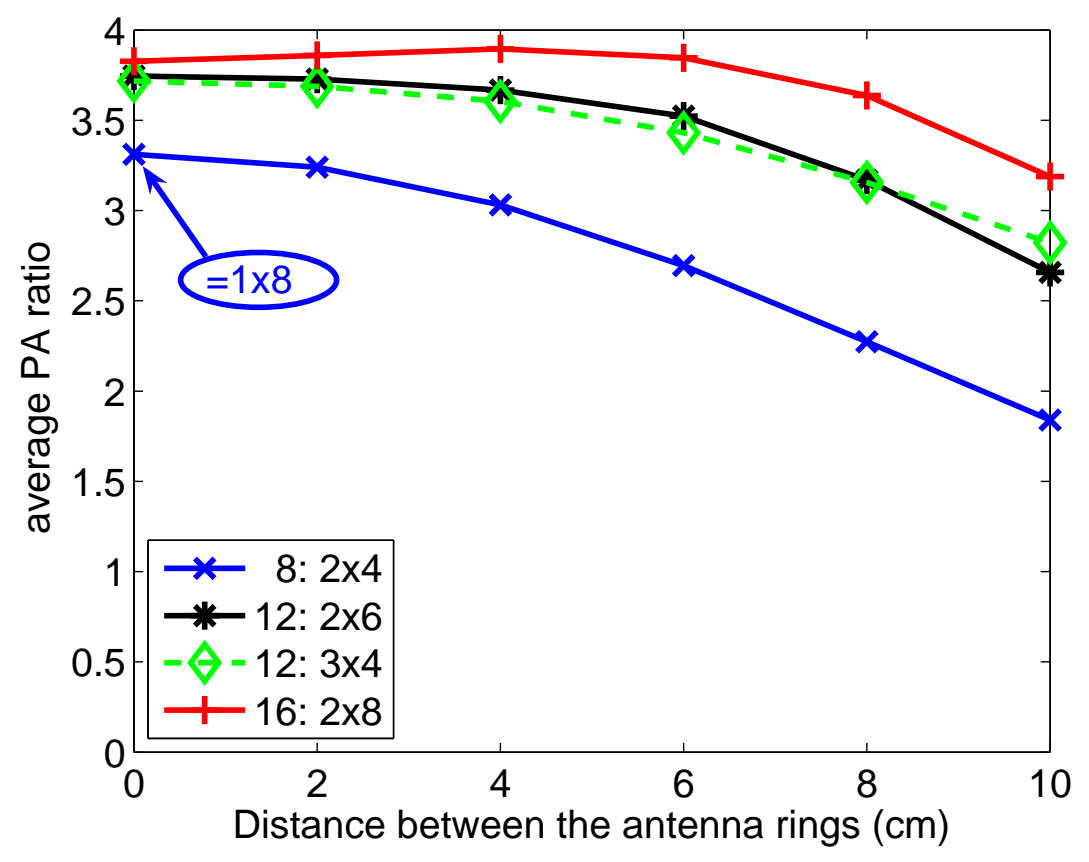

Large patient model

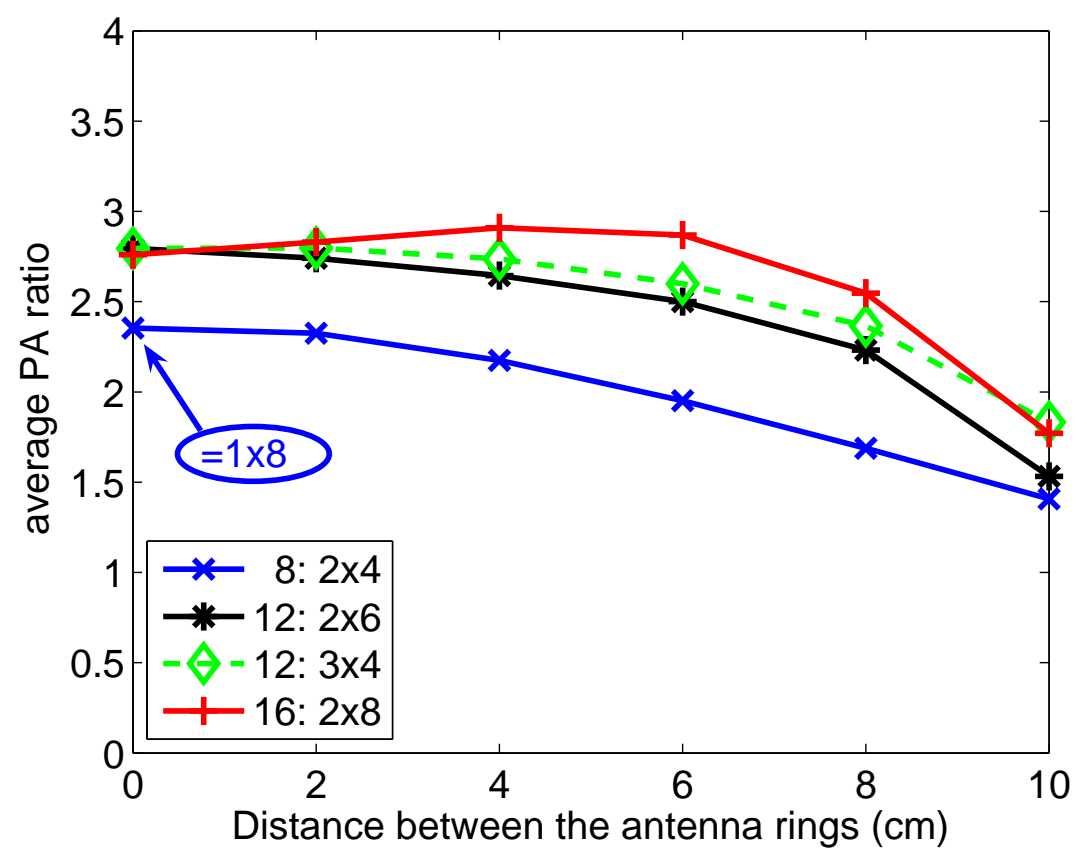

Figure 5: aPA ratios for different distances between the outer rings. Curves are shown corresponding to the $2 \times 4,2 \times 6,2 \times 8$ and $3 \times 4$ arrangements. 
with the total amount of antennas. For small distances $(<6 \mathrm{~cm})$ the aPA values indicate a $20 \%$ increase in focussing from eight to twelve antennas and an additional $10 \%$ increase for a 16 antenna setup.

\section{Discussion}

As we explained earlier [7], the causes of the perturbations on the SAR distribution of a phased array hyperthermia applicator device can be clustered in three groups. From inside to outside these are: 1) anatomy, 2) antenna array and 3) waterbolus related distortions. We chose to investigate these parameters independently to gain more understanding of their, sometimes opposing, effects on the PA pattern. In the present study we dedicated our attention to extending earlier work that has been done on step 2 [8]. Of course, it is difficult to separate waterbolus related influences from array related influences, especially at bolus-air transitions [15] [16]. However, waterbolus shapes are difficult to prescribe clinically and they highly depend on practical limitations. Further, effects from waterbolus edges that are present in many calculations due to straight edged models are expected to reduce due to more round shapes in the clinical practice. Therefore we chose to investigate antenna array effects and postpone studying effects of the waterbolus edges.

To perform the study within an acceptable period and to avoid an enormous amount of calculations, we simplified the problem by investigating only the ability of a circle symmetric antenna arrangement to adequately heat a centrally located target region. Exploiting the high amount of symmetry of this setup it was reasonable to use central phase and amplitude settings. Further, we assumed that the variation in aPA due to different patient anatomy locations is much larger than the variation due to suboptimal phase and amplitude settings. In a previous study [8] we have investigated the influence of the choice of frequency on the PA pattern by using one anatomy model. The frequency has a huge effect on the PA pattern (two fold increase in focussing) and for that study one anatomy model, and scaled versions, were sufficient to represent the entire group of $\mathrm{H} \& \mathrm{~N}$ patients that apply for RT. In this study we expected smaller differences and we therefore used two anatomy models that represented the extends of the subgroup of $\mathrm{H} \& \mathrm{~N}$ RT patients, i.e. a small patient and a large patient. The simplifications to the phase and amplitude optimization and anatomy models led to a considerable reduced computational effort while still a high predictive value for the optimum setup for this group of patients can be assumed. Phase and amplitude optimization, however, is expected to be inevitable to effectively steer the central focus to a non-central target region and is useful to further reduce hotspots per individual patient. These effects will be the subject of a subsequent study when the applicator is routinely used for clinical treatments giving us the opportunity to perform patient specific treatment planning. 
For this study we analysed CT scans of eight patients and selected the "most easy" and "most difficult" patient anatomy, i.e. a skinny patient with a long neck ("small patient model") and a fatty patient with a short neck ("large patient model"). We faced some difficulties to determine the optimum axial location of the array around the large patient's neck while trying to keep sufficient distance between all antennas and patient model's skin. In the first attempt large PA hotspots were visible, especially at the shoulders for setups with $\mathrm{D}>6 \mathrm{~cm}$. These hotspots where also reflected in steeper and less straightforward aPA curves. These problems, however, were less prominent in HT position compared to RT position. An improved placement, i.e. a shift in cranial direction, led to less hotspots and more predictable aPA curves.

This investigation is a follow-up of the study reported in a previous paper [8] and forms the step from that theoretical parameter study to an actual applicator antenna array setup. In that study we selected an array of six to eight antennas operating at $433 \mathrm{MHz}$ as optimum arrangement for a single ring, H\&N, HT applicator. In the present study we found that more than eight antennas yields better focussing, thus better aPA values. The reason for this different result is that by increasing the neck volume we now also incorporate the effects of PA hotspots at chin and shoulders that are reduced by the increased amount of antennas in the current study.

Earlier investigations used phased-array approaches to apply superficial hyperthermia with planar or conformal arrays [17] [18]. Experimental work by Gross et al. [19] showed the potential of cylindrical phased arrays for heating tumours in the neck using four waveguide horn antennas applied to a neck-sized cylindrical muscle-phantom. However, to the best of the authors knowledge, no clinical phased-array based applicator for applying hyperthermia to deep-seated head and neck tumours exists. Moreover, this is the first attempt to use 3D calculations for optimization of the parameters of a (circular) phasedarray applicator specifically designed for deep heating in the $\mathrm{H} \& \mathrm{~N}$ region.

For deep hyperthermia (DHT), the use of circular phased-array applicators has a long history [20] [21] and the application is to a certain extend comparable. A prominent difference that hampers comparison is the concave shape of the neck whereas the pelvis is more convex shaped. This concave shape results in more strict limitations on the distance between outer rings ( $\sim 8 \mathrm{~cm}$ as found in this study) where for DHT that dilemma is not that an important issue. Further, the neck has the advantage that it is more cylindrically symmetrical. As a consequence, SAR pattern optimization by phase steering is expected to be more straightforward.

The influence of multiple antenna rings for DHT was previously investigated theoretically by Seebass et al. [22], Paulsen et al. [23] and Kroeze et al. [24] for their deep hyperthermia systems consisting of three [22] [24] or four [23] rings of antennas at predefined positions. Paulsen et al. [23] conclude from their results (power based optimization) 
that increasing the number of antennas per ring in many cases is more favourable than increasing the number of antenna rings. A similar conclusion is drawn by Seebass et al. [22] for their power based optimization, but they found the reverse effect for the temperature based optimization. They explain this phenomenon by deviating between obtaining a proper focus at the target region with positive interference (power optimization) and the decrease of hotspots by negative interference (temperature optimization). Seebass et al [22] further conclude that a large amount of antennas per ring should be avoided because this results in a high sensitivity of the PA pattern to small amplitude and phase shifts. Kroeze et al. [24] compared a setups of multiple waveguide slot antennas for heating in the pelvic region. For this convex region they conclude that a $3 \times 4$ arrangement outperforms a $2 \times 6$ arrangement. Together these three studies agree with the current results that twelve antennas are sufficient for SAR pattern steering and that it is difficult to discriminate between a $3 \times 4$ and a $2 \times 6$ setup.

The influence of the distance between two arrays for deep HT is previously experimentally instigated by Crezee et al. [25]. They started with their AMC-4 setup, consisting of one ring of four waveguide antennas, and added an extra ring ("AMC-8") and varied the inner-ring distance. In contrast to our findings, they found by phantom measurements that the effective length $\left(\mathrm{L}_{50 \%}\right.$ : axial dimension of the $50 \%$ iso-SAR volume) increased for an increased ring distance. The reason for this difference is the relatively much smaller distance between the antennas and the patient for the AMC-8 system $(\sim 0.25 \lambda)$ compared to our setup $(\sim 1.5 \lambda)$. In a separate study in cylindrical phantoms (results not reported) we found that a small distance between the patient and the antennas results in a small

$\mathrm{L}_{50 \%}$ and thus a poor interference between the two antenna rings. In the extremum case of exposure of a muscle cylinder by a circumferential plane field, $\mathrm{L}_{50 \%}$ will reach $\infty$, providing the waves have sufficient penetration depth.

\section{Conclusions}

In this theoretical study we have focussed on investigating the dependence of the PA at the center of the neck as function of the arrangement of a multi-ring antenna array. Parameters of interest were: 1) patient positioning 2) antenna ring radius 3) number of antenna rings 4) number of antenna elements per antenna ring 5) distance between the outer antenna rings.

We found that a rotation of ten degrees of the antenna array with respect to the patient results in a better heating at depth $(\sim 11 \%)$. This rotation further aligns the spinal column to the central antenna array axis thus steering the central focus away from the spinal column by means of phase and amplitude adjustments is more straightforward. aPA values decreased $(\sim 21 \%)$ when increasing the antenna radius of a $1 \times 8$ setup. Mechanically, 
a radius of around $20 \mathrm{~cm}$ was experimentally found to fit best around the neck: thus we chose this smallest, practical, radius.

Investigations into an array with over eight antennas for this $\mathrm{H} \& \mathrm{~N}$ heating application revealed an increase of around $17 \%$ in aPA for $\mathrm{D}=0 \mathrm{~cm}$. It is difficult to chose the best performing setup $(2 \times 6$ or $3 \times 4)$ that matches our twelve, clinically available, amplifiers since only small differences in power focussing at depth are found. Furthermore, results are modulated by the array distance. Globally, the increase in penetration at depth of multiple rings diminished when the antenna rings where positioned at distances over $6 \mathrm{~cm}$. We chose to use two rings of six antennas because of its high intuitive steering (compared to a $3 \mathrm{x} 4$ setup) and an inner-ring distance of $6 \mathrm{~cm}$ for maximum axial (z-direction) SAR steering possibilities. Increasing our clinically available amount of amplifiers to $16(2 \times 8$ setup) would result in a $10 \%$ increase in focussing at depth when $\mathrm{D}=6 \mathrm{~cm}$.

\section{Acknowledgements}

This work is financially supported by the Maurits and Anna de Kock Foundation and the Dutch Cancer Society, grant DDHK 2003-2855. The authors further would like to thank M. Franckena for her segmentation work and J.B. Rhebergen for his help on fast simulations.

\section{References}

[1] El-Sayed S and Nelson N. Adjuvant and adjunctive chemotherapy in the management of squamous cell carcinoma of the head and neck region: a meta-analysis of prospective and randomized trials. J Clin Oncol 1996; 14: 838-847.

[2] Pignon JP, Bourhis J, Domenge C, et al. Chemotherapy added to locoregional treatment for head and neck squamous-cel carcinoma: three meta-analyses of updated individual data. Lancet 2000; 355: 949-955

[3] Trotti A. Toxicity in head and neck cancer: a review of trends and issues. Int $J$ Rad Onc Biol Phys 2000; 47: 1-12.

[4] Valdagni R., Liu F-F. and Kapp S. Important prognostic factors influencing outcome of combined radiation and hyperthermia, Int J Rad Onc Biol Phys 1988; 15: 959-972.

[5] Valdagni R. and Amichetti M. Report of long-term follow-up in a randomized trial comparing radiation therapy and radiation therapy plus hyperthermia to metastatic lymphnodes in stage IV head and neck patients. Int J Rad Onc Biol Phys 1993; 28: 163-169. 
[6] Wust P, Seebas M, Nadobny J, et al. Simulation studies promote technological development of radiofrequency phased array hyperthermia. Int J Hyperthermia 1996; 12: 477-498.

[7] M.M. Paulides, D.H.M. Wielheesen, J. Van der Zee, et al. Assessment of the local SAR distortion by major anatomical structures in a cylindrical neck phantom. Int $J$ Hyperthermia 2005; 21: 125-140.

[8] M.M. Paulides, S.H.J.A. Vossen, A.P.M. Zwamborn, et al. Theoretical investigation into the feasibility to deposit RF energy centrally in the head and neck region, Int $J$ Rad Onc Biol Phys 2005; 63: pp. 634-642.

[9] Haveman J, Van Der Zee J, Wondergem J, et al. Effects of hyperthermia on the peripheral nervous system: a review. Int J Hyperthermia 2004; 20: 371-392.

[10] Clemens CHM, Vossen SHJA, Woltering AB, et al. Study of the possible effects on health of the use of portable terminals within the C2000 radio network. TNO-FEL internal report: FEL-02-C152. 2003. Reproduction Department of The Netherlands Organisation of Applied Scientific Research (TNO), The Hague, The Netherlands.

[11] Van Leeuwen GMJ, Lagendijk JJW, Van Leersum BJAM, et al. Calculation of change in brain temperatures due to exposure to a mobile phone. Phys Med Biol 1999; 44: 2367-2379.

[12] Gabriel S, Lau RW, and Gabriel C. The dielectric properties of biological tissues: III. Parametric models for the dielectric spectrum of tissues. Phys Med Biol 1996; 41: 2271-2293.

[13] Stogryn A. Equations for Calculating the Dielectric Constant of Saline Water (Correspondence). IEEE Trans Micr Theor Tech 1971; 19: 733-736.

[14] Lagendijk JJW, Van den Berg PM, Hand JW, et al. Treatment planning and modelling in hyperthermia, a task group report. 1992, Postgraduate school of medical physics, II University of Rome.

[15] Hornsleth SN. Radiofrequency regional hyperthermia. PhD thesis, Aalborg University, 1996.

[16] de Bruijne M, Samaras T, Bakker JF, et al. Effects of waterbolus size, shape and configuration on the SAR distribution pattern of the Lucite cone applicator. Int $J$ Hyperthermia 2006; 22: 15-28. 
[17] Jouvie F, Bolomey JC, Gaboriaud G. Discussion of the capabilities of microwave phased arrays for hyperthermia treatment of neck tumours. IEEE Trans Micr Theor Tech 1986; 34: 495-501.

[18] Magin RL, Peterson AF. Noninvasive microwave phased arrays for local hyperthermia: a review. Int $J$ Hyperthermia 1989; 5: 429-450.

[19] Gross EJ, Cetas TC, Stauffer PR, et al. Experimental assessment of phased-array heating of neck tumours. Int J Hyperthermia 1990; 6: 453-474.

[20] Turner P. Apparatus for creating hyperthermia in tissue. US Patent No. 4,589,423. 1986.

[21] Van Dijk JD, Schneider C, Van Os R, et al. Results of deep body hyperthermia with large waveguide radiators. Adv Exp Med Biol 1990; 267: 315-319.

[22] Seebass M, Beck R, Gellerman J, et al. Electromagnetic phased arrays for regional hyperthermia: optimal frequency and antenna arrangement. Int J Hyperthermia 2001; 17: 321-336.

[23] Paulsen KD, Geimer S, Tang J, et al. Optimisation of pelvic heating rate distributions with electromagnetic phased arrays. Int J Hyperthermia 1999; 15: 157-186.

[24] Kroeze H, Van de Kamer JB, De Leeuw AAC, et al. Regional hyperthermia applicator design using FDTD modelling. Phys Med Biol 2001; 46: 1919-1935.

[25] Crezee J, Kok HP, Wiersma J, et al. Improving locoregional hyperthermia equipment using 3D power control: from AMC-4 to AMC-8. Abstracts ESHO-05, 2005: 14-15. 